\title{
Research on the Identification Method of Transmitter Recognition in CMMB Wireless Networks
}

\author{
Lei Guoping ${ }^{1, *}$, Xie Hong ${ }^{2}$, Qiu Gang ${ }^{1}$ and Dai Minlu ${ }^{1}$
}

\author{
${ }^{1}$ Key Laboratory of Signal and Information Processing, Chongqing Three Gorges University, Wanzhou,Chongqing, \\ 404100, P.R. China \\ ${ }^{2}$ Department of Mechanical and Electronic Engineering, Chongqing Three Gorges Vocational College, Chongqing, \\ 404155, P.R. China
}

\begin{abstract}
This papers designs the identification method of transmitter recognition in wireless networks by analyzing the wireless networks and the physical frame structure of China Mobile Multimedia Broadcasting(CMMB) system. The method calculates the time from the signal of preset transmitter to the measurement site according to the preset transmitter location information, the measured identification signal information of transmitter and the location information of measurement location. And then compare the time that the preset transmitter signal reaches the measurement site and that of the measured transmitter signal reaches the measurement site. Finally, it judges the preset transmitter identification corresponding to the concrete measured transmitter identification. The method can accurately recognize every transmitter when there are many transmitters with same autocorrelation value in the preset transmitter recognition, which is benefit for the optimizing of CMMB wireless networks.
\end{abstract}

Keywords: China Mobile Multimedia Broadcasting, CMMB, transmitter recognition, wireless networks, wireless network optimization.

\section{INTRODUCTION}

China Mobile Multimedia Broadcasting (CMMB) is the digital television terrestrial transmission standard introduced by broadcasting system of our country. It adopts the mobile television standard STiMi(Satellite and terrestrial interactive multi-service infrastructure) which is totally indigenous by our country. The standard is suitable for the broadcast frequency between $30 \mathrm{MHz}$ and $3000 \mathrm{MHz}$ and it can realize national roaming by the satellite and terrestrial wireless transmitting TV, broadcasting, data information and other multimedia signal broadcast system [1-3]. CMMB system apply two sets of receiving system which supports both $\mathrm{U}$ band (470 MHz 798 MHz) and S band (2635 $2660 \mathrm{MHZ}$ ) [4]. U band receives the signals from the terrestrial transmission network, while $\mathrm{S}$ band receives television and radio signals from satellites.

At present, CMMB network adopts Single-Frequency Network(SFN) hoc network. All the transmitters use the same frequency to translate the same signal at the same time in the SFN network, which makes it have good Space diversity gain [5]. The signal of CMMB SFN apply the orthogonal frequency division multiplexing (OFDM) technology [6]. There is a guard interval in front of each symbol in a frame,

*Address correspondence to this author at the Key Laboratory of Signal and Information Processing, Chongqing Three Gorges University, Wanzhou, Chongqing, 404100, P.R. China; Tel: +86 18323660286;

Fax: +86 23 58102309; E-mail: leiguoping@163.com which makes the system can ensure the orthogonality of subcarriers and prevent intercarrier interferences.

In CMMB system, the transmitter recognition signal is located in the beginning section of every time slot in the physical layer signal frame in order to identify different transmitters [7]. The transmitter recognition signal is the pseudorandom sequences whose length is 191 bits (physical layer bandwidth is $8 \mathrm{MHz}$ ) or 37 bits (physical layer bandwidth is $2 \mathrm{MHz}$ ) [8]. It has a total of 256 sequence, the sequence $0 \sim 127$ is used for the area identification, which identifies the transmitter location and transmits the even time slot inserted in the signal frame. The sequence 128 255 is the transmitter identification, which is used to identify different transmitters in the same area and transmit the odd slots inserted in the signal frame [9].

The physical frame structure of CMMB system is shown in Fig. (1). The physical layer signal is one frame per second, which is divided into 40 time slots. Every slot's length is $25 \mathrm{~ms}$, including 1 beacon and 53 OFDM symbols. Among them, the beacon structure includes the transmitter identification and two same synchronization signals $[10,11]$.

In the CMMB SFN system, recognizing every transmitter signal is one of the basic work in the digital television single frequency network optimization. The key of the transmitter signal recognition is to obtain various parameters of CMMB wireless network and the relative time of each transmitter reaches the measuring point. However, correctly identifying the transmitter recognition is the basic premise of identifying 


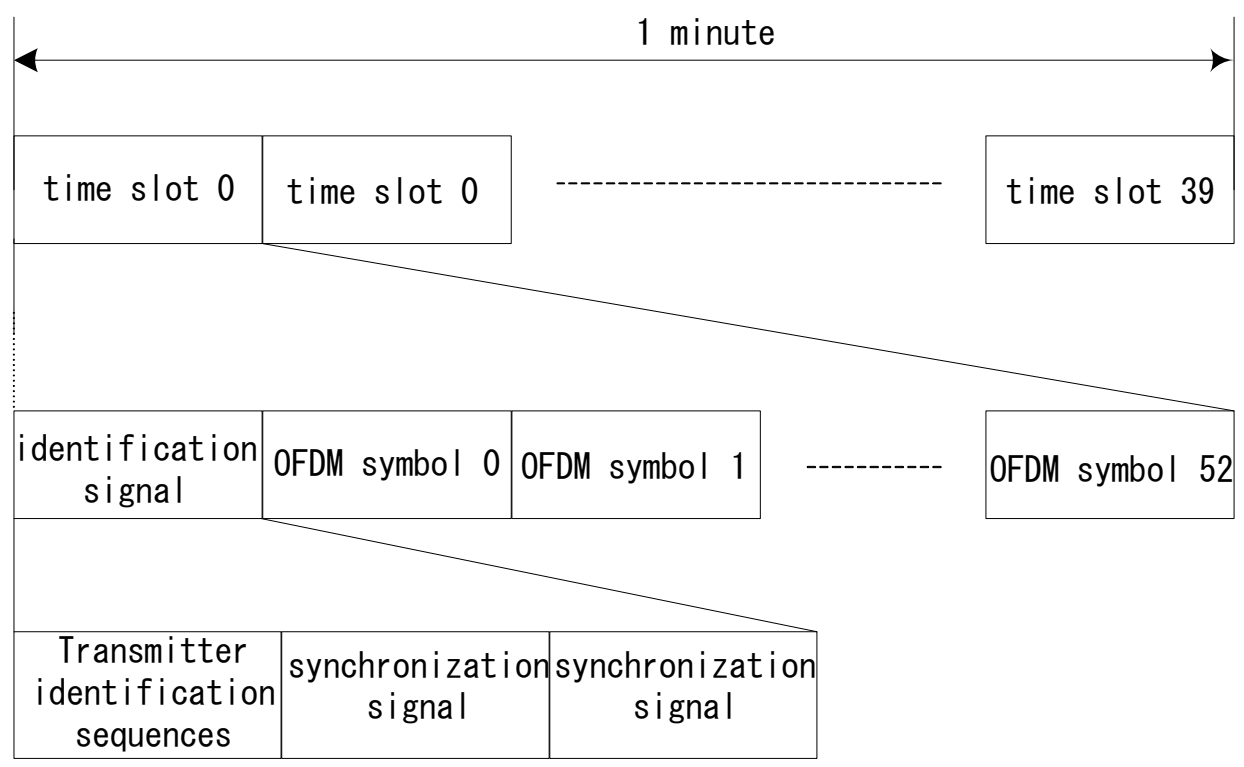

Fig. (1). CMMB physical layer frame structure.

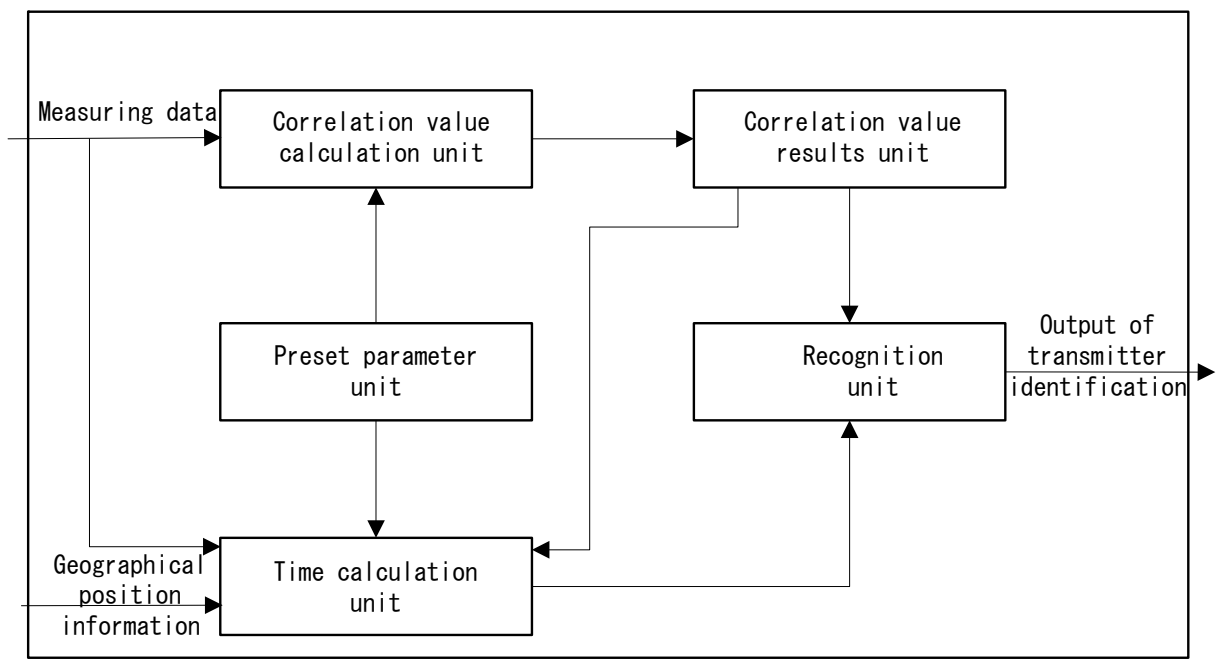

Fig. (2). Schematic diagram of CMMB wireless transmitter identification method.

every transmitter signal [12]. It is a basic way to make use of the orthogonality of transmitter recognition to identify every transmitter recognition, and then realize the identification of every transmitter signal. However, in the CMMB standard, not all the transmitter recognition is orthogonal. Therefore, it is difficult to accurately identify transmitter recognition according to the correlation value, which can make it possible to accurately identify which one is the transmitter of wireless signal in practical measurement. And then, it is difficult to realize the optimization of CMMB wireless network by using the practical measurement data $[13,14]$. Therefore, this paper proposes one method of transmitter recognition identification in CMMB wireless network in order to solve the problem of current technology that using the correlation value can not accurately identify the transmitter recognition, and then can not accurately identify the transmitter signal.

\section{THE DESIGN OF THE IDENTIFICATION METH- OD OF TRANSMITTER RECOGNITION}

The overall schematic diagram of the structure of CMMB wireless transmitter identification method is shown in Fig. (2).

It can know from Fig. (2), firstly, preset all the transmitter recognition of $\mathrm{CMMB}$ transmitter within the measured region, the geographical position information of transmitters and transmitters' parameters. And then receive the measuring data and carry out the correlation value calculation of preset transmitter recognition and the measured transmitter recognition to acquire the arrival time of the measured transmitter identification signal. Then calculate the arrival time of the preset transmitter signal which has the same correlation value calculation results of the measured transmitter 
signal form the preset location to the current location of transmitter according to the preset transmitter location, the current receiver location and the transmitter parameters. Finally, identify the preset transmitter recognition corresponding to the measured transmitter recognition signal according to the arrival time of the measured transmitter recognition signal and the preset transmitter recognition signal, namely regarding the preset transmitter recognition signal which has the nearest approximation arrival time as the measured transmitter recognition .

\subsection{Preset Parameter Unit}

Preset parameter unit, which is used to preset or store all the CMMB transmitter recognition, transmitter location information and transmitter parameters within the measured regions(province, city or district, country, etc.). The unit can actually corresponds to a storage medium which can both write and read, such as EPROM, memory chip or the magnetic storage media. On the other hand, the unit can make a contact with the correlation value calculation unit and preset parameter unit by the data bus and the corresponding readwrite control logic chip and control bus. When the preset transmitter launch with different transmitter frequency, the transmitter parameters should also include the parameters to calculate the time which the transmitter signal spend in transmitting in the network, such as the emission frequency, the location information which includes longitude, latitude and altitude.

\subsection{Correlation Value Calculation Unit}

Correlation value calculation unit makes a contact with the parameter subsystem of external wireless network by the external data and control bus and make a contact with preset parameters system and related results unit by the internal data and control bus, which is used to receive the measured data of the wireless network parameters measurement system's output, execute the correlation value calculation between the preset transmitter recognition and the measured transmitter recognition, and transmit the resulted data of the correlation value calculation output to the related results unit to save. The unit can actually corresponds a hardware chip composed of an input/output logic and the correlation value calculation. Preset transmitter recognition is read from the preset parameter unit and the measured transmitter recognition is obtained from the resolution from the measured data input interface. The resulted data of the correlation value calculation should at least include the measured transmitter recognition, the preset transmitter recognition which has the same correlation value with the measured transmitter recognition and the corresponding correlation value.

In the CMMB wireless network system, it can have 256 transmitter recognitions. $R_{i}$ stands the related calculation value of the transmitter recognition $i$. The measured transmitter recognition signal is $m . m$ is a one-dimensional vector[15]. The transmitter recognition specified in the CMMB standard namely preset transmitter recognition is $s, s$ is a one-dimensional vector. Therefore:
$R_{i}=\sum\left(m_{j} \otimes s_{i j}\right)$

Among the equation, $i$ is used to identify the serial number of the transmitter recognition defined in the CMMB wireless network system.

\subsection{Correlation Value Results Unit}

The correlation value results unit is used to cache the results of correlation value calculation. The resulted data of the correlation value calculation should at least include the measured transmitter recognition, the preset transmitter recognition which has the same correlation value with the measured transmitter recognition and the corresponding correlation value.

The correlation value calculation unit makes a contact with the correlation value calculation unit, the recognition unit and the time calculation unit by the data or control bus, which is used to cache the resulted data of correlation value calculation which is sent by the correlation value calculation unit. The unit can actually correspond to a storage medium, such as random access memory RAM.

Suppose that there are 256 transmitter recognitions in the preset parameter unit. but since not all the CMMB transmitter recognition is orthogonal sequence, there are several transmitter recognition with the same correlation value in the preset transmitter recognition, which can lead to the measured transmitter recognition which is calculated by the correlation value calculation unit have exactly the same calculation results of the correlation value calculation with the preset value transmitter recognition or within the preset error range (as for a concise representation, this paper collectively call them the same correlation value), which makes it is difficult to distinguish which preset transmitter recognition is corresponding to the measured transmitter recognition with the same correlation value. Of course, if the correlation value of the measured transmitter recognition is not the same with all the preset transmitter recognition (or not in the within the preset deviation range), the preset transmitter recognition with the highest correlation can be directly determined the measured transmitter recognition. In this paper, it solves the problem mentioned above that it is difficult to identify the measured transmitter recognition when the correlation is the same by the combination of time calculation unit, recognition unit and the location information of transmitter and receiver.

\subsubsection{Time Calculation Unit}

Time calculation unit have a contact with parameter subsystem of the external wireless network, preset parameter unit and correlation results unit, which is used to calculate the correlation value calculation results to find out the preset transmitter recognition signal which has the same correlation value with the measure transmitter recognition and the time of preset transmitter recognition spend on the transmitting from the preset location to the receiver location and the arrival time of measured transmitter recognition. 
The time calculation unit includes the first calculation unit and the second calculation unit. The first calculation unit presets the parameter unit and connect the correlation unit and is used to get the preset transmitter recognition with the same correlation value of the measured transmitter recognition among the correlation value result data from the correlation value result unit. And then, find the transmitter parameter and the location information corresponding to the preset transmitter recognition from the preset parameter unit according to the preset transmitter recognition read from the correlation result unit. Then calculate the arrival time of preset transmitter recognition from the preset place to the receiver location according to the location information of receiver and send the calculated arrival time of every preset transmitter recognition to the recognition unit. The unit can actually be realized by the input/output logic and computational logic chip.

The second calculation unit of the time calculation unit connect with the parameter subsystem of the external wireless network, which is used to calculate the arrival time of the measured transmitter recognition signal and send the calculated results to the recognition unit.

The unit make a contact with the parameter subsystem of the external wireless network by the external data and control bus and make a contact with the preset parameter unit, correlation value result unit and recognition unit through the internal data and control bus. It is used to the calculate the arrival time of preset transmitter recognition with the same correlation value of measured transmitter recognition among the correlation calculation results from the preset location to the receiver location and the arrival time of the measured transmitter recognition.

The second calculating unit obtain the arrival time of the measured transmitter recognition according to the measured data obtained from the parameter subsystem of the external wireless network. The arrival time of the measured transmitter recognition is the time from the transmitter to the receiver of the measured transmitter recognition which is calculated according to the transmission parameters of the wireless transmission channel corresponding to the measured transmitter recognition or obtained according to the time difference between the time the transmitter launch the signal in the wireless transmission channel and the time the receiver receives the signal. The unit stores every measured transmitter recognition and its signal arrival time to the receiver location. The unit can actually be realized by the input/output logic and computational logic chip.

The specific details to obtain the arrival time of the preset transmitter recognition are as follows: firstly, calculate the signal transmission distance according to the geographical location of the preset transmitter recognition parameters and the current location of receiver. And then make the Simulation calculation of the arrival time of the preset transmitter from the preset location to the current location of receiver according to the parameters like signal transmitting frequency of the preset transmitter parameters.

The specific details to obtain the arrival time of the preset transmitter recognition are as follows: firstly, calculate the signal transmission distance according to the geographical location of the preset transmitter recognition parameters and the current location of receiver. And then make the Simulation calculation of the arrival time of the preset transmitter from the preset location to the current location of receiver according to the parameters like signal transmitting frequency of the preset transmitter parameters.

\subsubsection{Recognition Unit}

The recognition unit connects with the correlation results unit and time calculation unit. It is used to recognize the preset transmitter recognition actually corresponding to the measured transmitter recognition according to the arrival time of the measured transmitter recognition and the preset transmitter recognition and export recognized transmitter recognition.

Through the connecting of the data and control bus, the correlation results unit and time calculation unit, it is used to recognize the preset transmitter recognition actually corresponding to the measured transmitter recognition according to the arrival time of the measured transmitter recognition and the preset transmitter recognition and export recognized transmitter recognition and then recognized the preset transmitter recognition which has the closest correlation value of the measured transmitter recognition as the measured transmitter recognition and export recognized transmitter recognition. When there is no preset transmitter recognition which has the same correlation value with the measured transmitter recognition(or all are out of the preset deviation range),the unit will recognize the preset transmitter recognition which is in the highest correlation of the measured transmitter recognition as the measured transmitter recognition and outputs the recognized transmitter recognition. The unit can be achieved by the input / output logic and comparator.

\section{EXPERIMENTAL RESULTS AND ANALYSIS}

According to the transmitter recognition and the geographical location information corresponding to the transmitter and based on the correlation detection for transmitter recognition, as for the transmitter recognition which can not be detected by the correlation detection, we can calculate the time of preset transmitter signal reaches the measurement region according to the geographical location of preset transmitter recognition, the measured transmitter recognition and measurement location. And then compare the arrival time of the preset transmitter recognition and the measured transmitter recognition to the measurement location and determine which preset transmitter recognition is corresponding to the measured transmitter recognition.

Fig. (3) is the structure schematic diagram of CMMB wireless network analyzer used in the road test, it consists of two main parts in the internal CMMB wireless network analyzer namely the wireless network parameter measurement subsystem and transmitter recognition measurement and analysis subsystem respectively. Among them, the wireless network parameter measurement system will measure data and send them to the transmitter recognition measurement and analysis subsystem, and then the transmitter recognition measurement and analysis subsystem will recognize the transmitter recognition and give the relative time delay of 


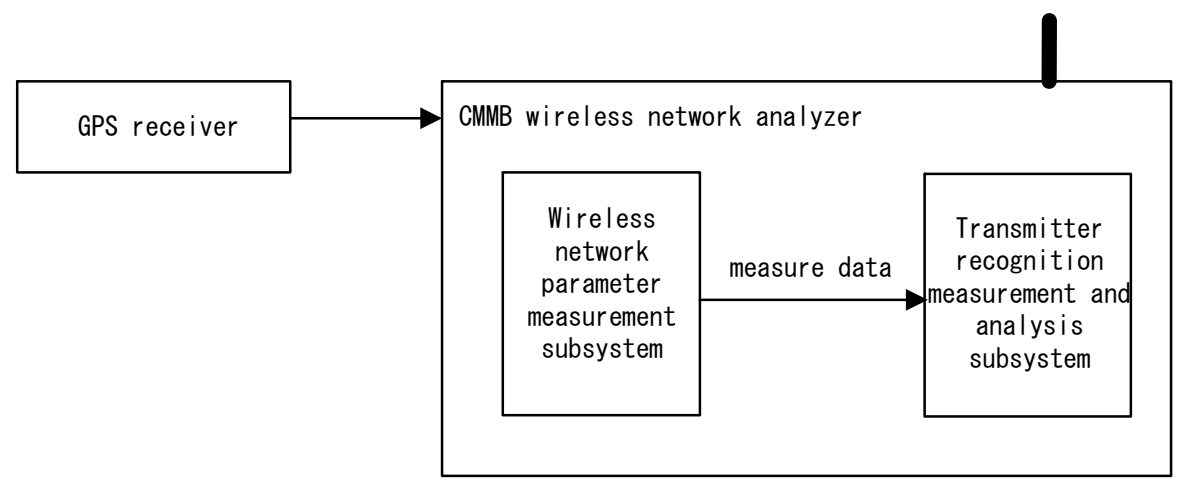

Fig. (3). The structure schematic diagram of the CMMB wireless network analyzer.

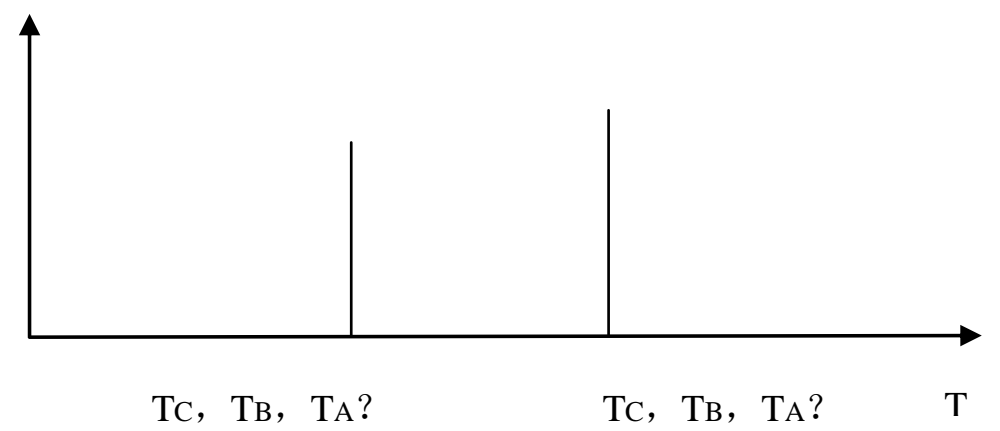

Fig. (4). The sketch map of the arrival time and amplitude of two measured transmitter measured in current measurement location.

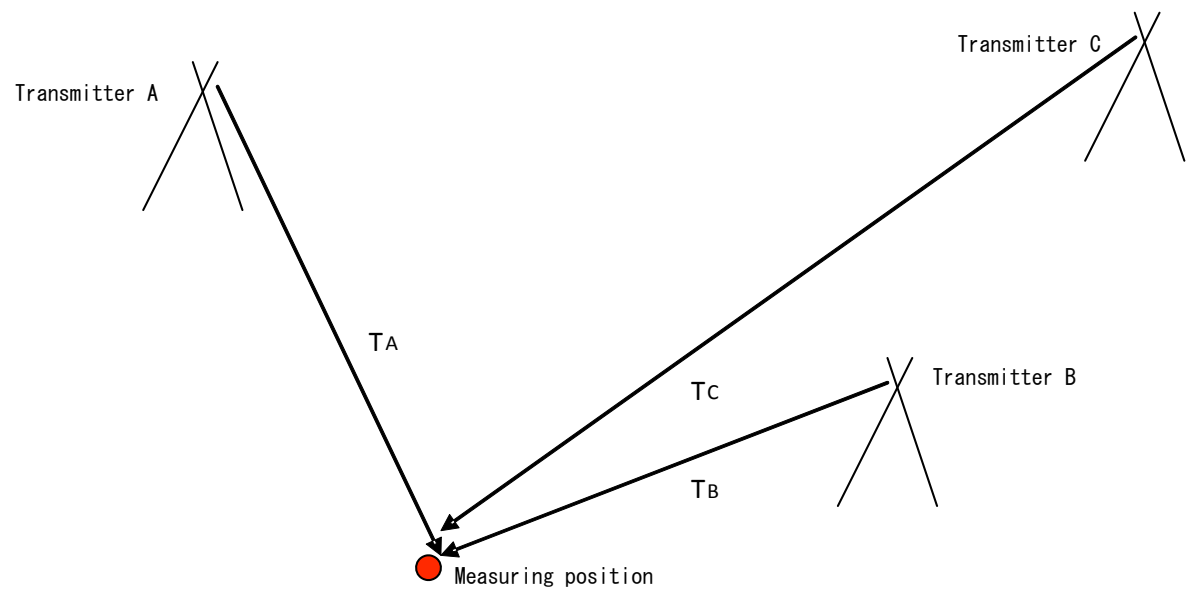

Fig. (5). The sketch map of the positional relation between the three transmitters A, B, C and the measurement position.

transmitter signal. The measured data should include but not limited to the measured transmitter recognition and its arrival time.

Suppose that the number of the measured transmitter recognition in correlation results unit is less than that of the preset transmitter recognition in the preset parameter unit. Suppose that there are three preset transmitter A,B,C and its geographical location information. The transmitter autocorrelation values of $A, B, C$ are the same.

Fig. (4) is an example of the arrival time and amplitude of two measured transmitter measured in current measurement location. The abscissa is the time axis and the ordinate is the amplitude of the signal. After the calculation of corre- lation value calculation unit, we found that there are two measured transmitter recognition has the same correlation value of three preset the transmitter recognition in the preset parameters unit. So the correlation value cannot distinguish the two transmitters through the two measured transmitter recognition.

Time calculation unit calculates the arrival time that the preset transmitter recognition signal reaches the measurement location according to the preset geographical location information of the three preset transmitter recognitions with the same correlation value and the geographical location information of the receiver. Fig. (5) is an example of the positional relation between the three transmitters $\mathrm{A}, \mathrm{B}, \mathrm{C}$ and 


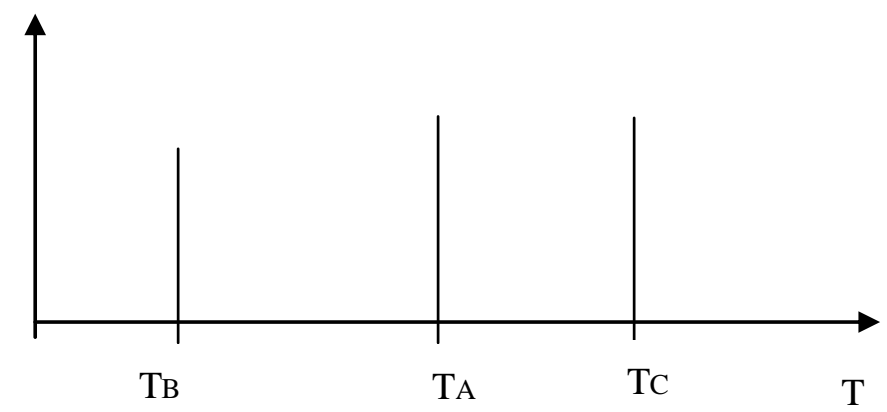

Fig. (6). The relationship of amplitude and time that the three preset transmitters' signal reach the measuring position.

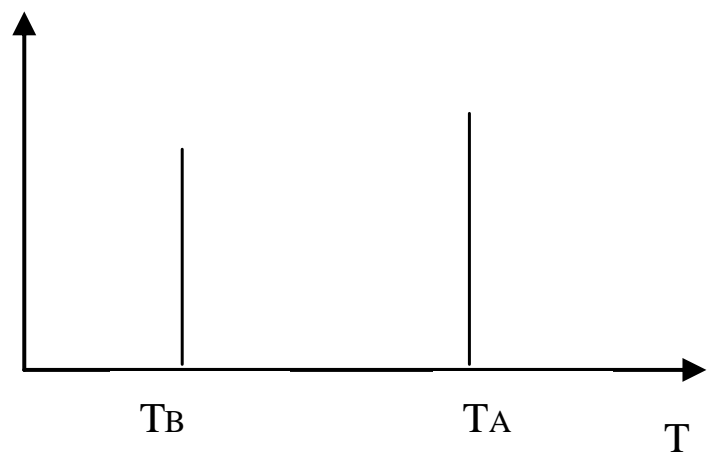

Fig. (7). The relationship of two measured transmitters and two preset transmitters.

the measurement position. $T_{\mathrm{A}}$ is the arrival time of the preset transmitter A's recognition signal from the preset position to the receiver location, namely the arrival time of the preset transmitter A's recognition signal in current measurement position. $\mathrm{T}_{\mathrm{B}}$ is the arrival time of the preset transmitter B's recognition signal from the preset position to the receiver location, namely the arrival time of the preset transmitter B's recognition signal in current measurement position. $T_{c}$ is the arrival time of the preset transmitter C's recognition signal from the preset position to the receiver location, namely the arrival time of the preset transmitter C's recognition signal in current measurement position.

Fig. (6) is the sketch map of the arrival time and amplitude of the three signals of the transmitters A, B, C reach the measurement position. Because the measurement position is closer to the transmitter B, so the transmitter B's signal firstly arrived at the measurement location. While the measurement location is most far away from the transmitter $\mathrm{C}$, so the transmitter C's signal is the latest one reach the measurement position.

According to time calculating unit's computing results as well as correlation value result unit's calculating results, recognition unit can match the measured transmitter with two of the preset transmitter A, B, C.

Correlation value result unit measures two time parameter that transmitter signals reach the measuring location. Time calculating unit computers the time that the transmitter $\mathrm{A}$, transmitter $\mathrm{B}$ and transmitter $\mathrm{C}$ reach the measure position. Recognition unit compares the time one by one and find two results. The arrival time of transmitter B is the closest to the first transmitter's arrival time. The arrival time of transmitter A is the closest to the second transmitter's arrival time. So we declare that the first transmitter is transmitter B and the second transmitter is transmitter A. As for the far distance, transmitter C's power attenuation is so serious that signal is too weak to meet the threshold value as well as be detected when it reach the receiver. The matched transmitter and time-delay of signal are shown as Fig. (7).

Assume that the recognition numbers of transmitters which are measured by relevant values result unit are equal to the identify numbers of transmitters which are preset by parameters preset unit. On the condition that transmitter A, B and C's geographic information are preset by parameters preset unit, and the three parameters have the same autocorrelation function value. The relationship of transmitter A,B,C's location is shown as Fig. (5).

The three transmitter signals' arrival time as well as amplitude which is measured in the current detective position is shown as Fig. (8). Through the calculating of relevant values result unit, we find that the measured three identify number of transmitters are equal to the identify number of transmitters which are preset by parameters preset unit.

According to the current location, time calculating unit computer the time that each signal take to reach transmitters location, the relationship of signal amplitude as well as time is shown as Fig. (9). $T_{A}$ means the arrival time that recognition signal of transmit A from preset location to receiver, namely, the arrival time of transmitter A's recognition 


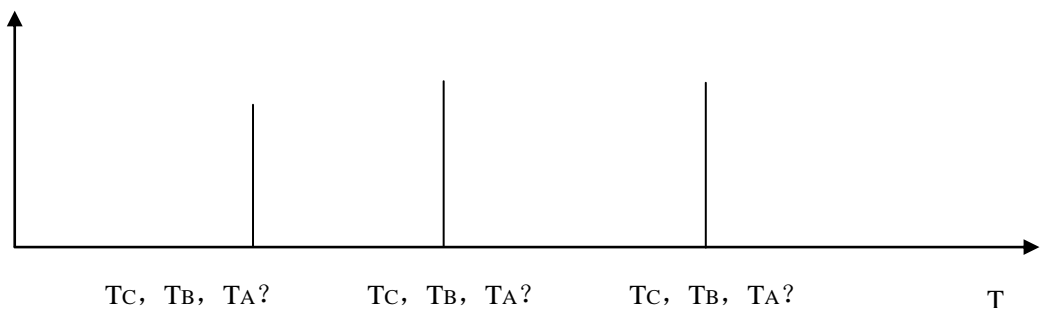

Fig. (8). The relationship of the measured three transmitter signals' arrival time as well as amplitude.

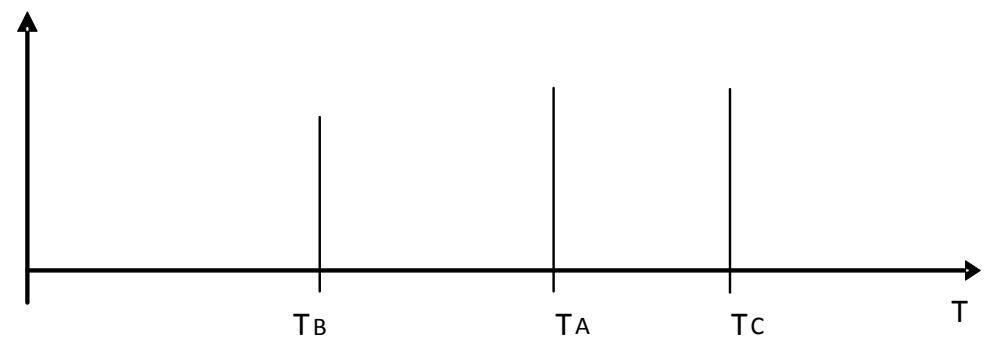

Fig. (9). The relationship of the three preset transmitter signals amplitude as well as time.

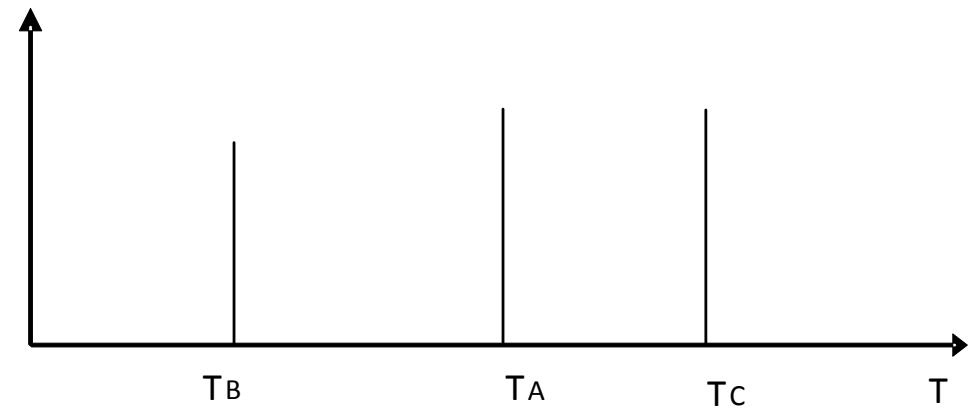

Fig. (10). The relationship of measured transmitter and the preset transmitter.

signal. $T_{B}$ means the arrival time that recognition signal of transmit $\mathrm{B}$ from preset location to receiver, namely, the arrival time of transmitter $B$ 's recognition signal. $T_{C}$ means the arrival time that recognition signal of transmit $\mathrm{C}$ from preset location to receiver, namely, the arrival time of transmitter C's recognition signal. Since transmitter B is the nearest to the measure location, signal of transmitter $\mathrm{B}$ reach detecting position at first. Transmitter $\mathrm{C}$ is the furthest to the measure position, so signal of transmitter $\mathrm{C}$ reach detecting position at last.

Recognition unit compares the time that measured by relevant values result unit with the time that calculated by time calculating unit one by one and find that, the reaching time of transmitter B is the closest to the time that the first transmitter reach detect position, the reaching time of transmitter $\mathrm{A}$ is the closest to the time that the second transmitter reach detect position, the reaching time of transmitter $\mathrm{C}$ is the closest to the time that the third transmitter reach detect position. So we declare that the first measured transmitter is transmitter $\mathrm{B}$, the second measured transmitter is transmitter $\mathrm{A}$, the third measured transmitter is transmitter $\mathrm{C}$, as shown in Fig. (10).

\section{CONCLUSION}

The identification method of transmitter recognition include preset parameter unit, correlation value calculation unit, correlation value results unit, time calculation unit and recognition unit.

Preset parameter unit is used to store all the CMMB transmitter recognition, transmitter location information and transmitter parameters within the measured regions.

Correlation value calculation unit parameter, which is connected with subsystem of external wireless network as well as internal preset data unit, is used to carry out the correlation value calculation of preset transmitter recognition and the measured transmitter recognition, to transmit the resulted data of the correlation value calculation output to the related results unit.

Correlation value results unit is used to cache the results of correlation value calculation. The resulted data of the correlation value calculation should at least include the measured transmitter recognition, the preset transmitter recognition which has the same correlation value with the measured 
transmitter recognition and the corresponding correlation value.

Time calculation unit, which is contacting with parameter subsystem of the external wireless network, preset parameter unit and correlation results unit, is used to calculate the correlation value calculation results to find out the preset transmitter recognition signal which has the same correlation value with the measure transmitter recognition and the time of preset transmitter recognition spend on the transmitting from the preset location to the receiver location and the arrival time of measured transmitter recognition.

Recognition unit, which is connecting with the correlation results unit and time calculation unit, it is used to recognize the preset transmitter recognition actually corresponding to the measured transmitter recognition according to the arrival time of the measured transmitter recognition and the preset transmitter recognition and export recognized transmitter recognition.

The algorithm to identification transmitters of CMMB single-frequency network, which is first proposed in the area of mobile digital television, is a simple and practicable algorithm and provide valuable basic data for further study on optimization of the CMMB wireless network system.

\section{ABOUT THE AUTHORS}

First Author Lei Guoping, University lecturer of Chongqing Three Gorges University. The author's major is Mobile digital TV and Communication Technology. 6 papers received by EI and 2 patents had been published.

Second Author Xie Hong, master degree in engineering. The author's major is Mobile digital TV.

Third Author Qiu Gang, master degree in engineering. The author's major is Electronic Information Technology.

Fourth Author Dai Minlu, University professor of Chongqing Three Gorges University, Ph.D. The author's major is Mobile digital TV and Communication Technology.

\section{CONFLICT OF INTEREST}

The authors confirm that this article content has no conflict of interest.

\section{ACKNOWLEDGEMENTS}

This work was financially supported by Program for Innovation Team Building at Institutions of Higher Education in Chongqing(Grant No.KJTD201320); Project Supported by Achievement Transfer Program of Institutions of Higher Education in Chongqing; Project Supported by Scientific and Technological Research Program of Chongqing Municipal Education Commission (Grant KJ131118) ; Project Supported by Open fund of Chongqing Three Gorges University of Key Laboratory of Signal and Information Processing.

\section{REFERENCES}

[1] CMMB research teams, GY/ T220, 2-2006 of Radio, Film and TV industry in China, CMMB multiplexing, 2006. (In Chinese).

[2] CMMB research teams. Part II: multiplex implementation guidelines, 2007 (In Chinese).

[3] Q. Yang, T. Tao, and G. Qihong, GY/T. 220.2-2006. Mobile Multimedia Broadcasting Part 1: Framing Structure, Channel Coding and Modulation for Broadcasting Channel, Beijing, 2006 (In Chinese).

[4] X. Wei, X. Guo, and Q. Yang, GY/T. 220.2-2006. Mobile Multimedia Broadcasting Part 2: Multiplexing, Beijing, 2006 (In Chinese).

[5] Y. Wang, J. H. Ge, J. Hu, and B. Ai, "An efficient OFDM timing synchronization for CMMB system", IEICE Trans. Commun., vol. E95-B, pp. 3786-3792, 2012.

[6] J. Liao, and X. Yang, "A handover algorithm in the converged networks of CMMB and TD-MBMS", Int. J. Internet Protocol Tech., vol. 8, pp. 33-43, 2014.

[7] X. Wei, X. Guo, and G. Fu, GY/Z 234-2008. Implementation guide for mobile multimedia broadcasting multiplexing, Beijing, 2008 (In Chinese).

[8] G. P. Lei, and X. Y. Yineng, "The design and implementation of multiple services' tracking and parallelly testing in CMMB system", Appl. Mech. Mat., vol. 427-429, pp. 506-510, 2013.

[9] X. Wan, J. Yia, Z. Zhao, and H. Ke, "Experimental research for CMMB-based passive radar under a multipath environment", IEEE Trans. Aerospace Electron. Syst., vol. 50, pp. 70-85, 2014

[10] R. Wang, "System description of the CMMB terrestrial delivery system", ABU Tech. Rev., vol. 251, pp. 8-11, 2012

[11] X. R. Wan, B. Cen, J. X. Yi, L. Fang, and H. Y. Ke, "Reference signal extraction methods for CMMB-based passive bistatic radar", $J$. Electro. Inform. Tech., vol. 34, pp. 338-343, 2012. (In Chinese)

[12] T. Yang, "Novel 4M-mode for SOFDM transmitting in CMMB system”, Chin. Space Sci. Tech., vol. 32, pp. 42-50, 2012. (In Chinese)

[13] M. Yan, X. G. Wang, and J. F. Li, "Research and design on China mobile multimedia broadcasting network planning", Appl. Mech. Mat., vol. 536-537, pp. 726-730, 2014

[14] M. Yan, X. G. Wang, and J. F. Li, "3D video transmission system for china mobile multimedia broadcasting", Appl. Mech. Mat., vol. 519520, pp. 467-470, 2014

Received: September 22, 2014

Revised: November 03, 2014

Accepted: November 06, 2014

(C) Guoping et al.; Licensee Bentham Open.

This is an open access article licensed under the terms of the Creative Commons Attribution Non-Commercial License (http://creativecommons.org/licenses/by-nc/3.0/) which permits unrestricted, non-commercial use, distribution and reproduction in any medium, provided the work is properly cited. 